\title{
The Austrian ICU survey
}

\section{A questionnaire-based evaluation of intensive care medicine in Austria}

\author{
Christine Schlömmer · Gregor A. Schittek · Jens Meier · Walter Hasibeder · Andreas Valentin · Martin W. Dünser
}

Received: 4 May 2021 / Accepted: 27 December 2021 / Published online: 27 January 2022

(c) The Author(s), under exclusive licence to Springer-Verlag GmbH Austria, part of Springer Nature 2022

\begin{abstract}
Summary
Background While structures of intensive care medicine in Austria are well defined, data on organisational and medical practice in intensive care units (ICUs) have not been systematically evaluated.

Methods In this explorative survey, organisational and medical details of ICUs in Austria were collected using an online questionnaire consisting of 147 questions. Results Out of 249 registered ICUs 73 (29.3\%) responded, 60 were adult, 10 pediatric/neonatal ICUs and 19, 25 and 16 ICUs were located in level I, II and III hospitals, respectively. Of the respondents $89 \%$ reported that the ICU director was board-certified in intensive care medicine. Consultants were constantly present in $78 \%$ of ICUs during routine working hours and in $45 \%$ during nights and weekends. The
\end{abstract}

Supplementary Information The online version of this article (https://doi.org/10.1007/s00508-021-02002-x) contains supplementary material, which is available to authorized users.

\section{Schlömmer}

Department of Anesthesia, Critical Care and Pain Medicine, Medical University Vienna, Vienna, Austria

\section{G. A. Schittek}

Department of Anesthesiology and Intensive, Medical University Graz, Graz, Austria

J. Meier · OA PD Dr. M. W. Dünser, DESA EDIC ( $₫)$ Department of Anesthesiology and Critical Care Medicine, Kepler University Hospital and Johannes Kepler University Linz, Krankenhausstraße 9, 4020 Linz, Austria

martin.duenser@kepleruniklinikum.at

W. Hasibeder

Department of Anaesthesiology and Critical Care Medicine, Hospital Zams, Zams, Austria

\section{A. Valentin}

Department of Internal and Critical Care Medicine, Hospital Schwarzach, Schwarzach, Austria nurse:bed ratio varied between 1:1 and 1:2 in $74 \%$ during day shifts and $60 \%$ during night shifts. Routine physiotherapist rounds were reported to take place daily except weekends in $67 \%$ of ICUs. Common monitoring techniques were reported to be in routine or occasional use in $85 \%$ and $83 \%$ of ICUs, respectively. The majority of ICUs provided daily visiting hours ranging between $2-12 \mathrm{~h}$. Waiting rooms for relatives were available in $66 \%$ and an electronic documentation system in $66 \%$ of ICUs. Written protocols were available in $70 \%$ of ICUs.

Conclusion The Austrian ICU survey suggests that ICUs in Austria are clearly structured, well-organized and well-equipped and have a high nurse:bed ratio. In view of the relatively low return rate we cannot exclude that a selection bias has led to overestimation of the survey findings.

Keywords Intensive care units - Austria - Structure Organization · Equipment

\section{Introduction}

Intensive care medicine is a specialized field in medicine and practised by representatives of different medical specialties in Austria. Internationally, Austria ranks high in terms of availability of intensive care unit (ICU) beds [1] as well as the quality of intensive care treatment [2]. Structures of intensive care medicine in Austria are well defined. Before the coronavirus disease 2019 (COVID-19) pandemic, approximately 2500 ICU beds were available in 177 Austrian hospitals [3]. Adult ICUs in Austria are categorized into four levels (intermediate care units, levels 1-3 ICUs) for which clear requirements regarding staffing as well as availability of monitoring and therapeutic equipment have been outlined [4]. Pediatric and neonatal ICUs are dedicated to the care of critically ill 
children $<15$ years and during the first 28 days of life, respectively. Similar to adult ICUs, specific requirements for staffing and equipment of pediatric ICUs have been issued [4]. Financial reimbursement of intensive care services is performed according to the Performance-oriented Hospital Financing (leistungsorientierte Krankenanstaltenfinanzierung) and the Austrian Health Care Structure Plan (Österreichischer Strukturplan Gesundheit); however, these plans lack clearly defined requirements for each ICU category and intermediate care, i.e. minimum nurse:bed ratio [5]. Despite the availability of these and other structural information on the Austrian ICU landscape, data on organizational and medical practice in Austrian ICUs (e.g., shift patterns, inclusion of other medical specialties and allied healthcare specialists into ICU rounds, frequency of equipment use, availability of special organ support therapy and standard operating procedures, quality indicators, patient and family care) have not yet been systematically evaluated.

In this explorative, questionnaire-based survey, we sought to collect organizational and medical details of daily practice in Austrian ICUs and separately report these for adult ICUs, as per their location in different hospital levels, and pediatric ICUs.

\section{Material and methods}

This study was designed as an explorative web-based, prospective, cross-sectional, self-reported questionnaire-based survey among Austrian ICUs and was conducted under the auspices of the Federation of the Austrian Societies of Intensive Care Medicine (FASIM). Data acquisition took place from 16 January until 12 March 2020, when the survey was prematurely terminated due to the escalating COVID-19 pandemic in Austria. Since the survey was based on voluntary participation and information disclosure, the study protocol did not undergo review by an ethics committee.

\section{Surveyed ICUs}

All units registered as adult ICUs of the levels 1-3 (as defined by the Austrian Health Care Strucutre Plan (Österreichischer Strukturplan Gesundheit [4]): level 1 ICUs provide basic intensive care whereas level 3 ICUs have equipment available to provide all evidence-based organ support techniques) as well as all pediatric including neonatal ICUs were eligible for participation in this survey. We did not include intermediate care units in our survey. Electronic letters of invitation were sent through the FASIM office to the chair of each department running an ICU. Each letter of invitation included a link to the web-based questionnaire at www.surveymonkey.com. In case two or more ICUs were affiliated with one department, the department chair was asked that one questionnaire was completed per ICU. One reminding note was sent.

\section{Study questionnaire and data processing}

The study questionnaire consisted of 147 questions, was grouped into 5 sections and could be downloaded from the electronic repository. The study questionnaire was available in German, the official language of Austria. It underwent pilot testing by the study investigators with respect to flow, salience, acceptability and administrative ease. Open-ended questions were reduced to a minimum and multiple answers were only allowed for those questions where this was considered absolutely necessary. Based on the results of the pilot testing, the questionnaire was modified and finally approved by all investigators.

The first section of the questionnaire retrieved general information on characteristics and staffing patterns of the surveyed ICU and hospital. In detail, the level of hospital care was recorded. According to the Austrian Health Care Strucutre Plan [4], level I hospitals correspond to primary care hospitals (e.g. regional hospitals), level II hospitals to secondary care hospitals (e.g. referral hospitals) and level III hospitals to tertiary care hospitals (e.g. university teaching hospital). In order to guarantee anonymity of the respondents, we did not collect information on the level of care of the ICU. Section two collected data on the availability and frequency of use of monitoring techniques and diagnostic equipment. Section three included questions on the spectrum and frequency of use of therapeutic options available in the ICU and hospital of the respondent. While section four focused on quality indicators in the surveyed ICUs, the last section of the questionnaire recorded information on patient and family care.

After online completion of the questionnaire, data were saved and automatically transferred into a spreadsheet. At the end of the survey period, questionnaire accessibility through the study homepage was blocked and raw data were manually and independently checked by two authors for plausibility and quality control. Open-ended questions in the database were numerically coded.

\section{Study objectives and statistical analysis}

The primary objective of this study was to explore organizational and medical details of daily practice in Austrian ICUs. The secondary study objective was to separately report primary objectives for ICUs located in different levels of hospitals as well as pediatric/ neonatal ICUs.

The SPSS software program was used for data analysis (SPSS 15.0; SPSS Inc, Chicago, IL, USA). Descriptive methods were used to report absolute numbers with percentages for binary study variables and me- 
Table 1 Characteristics of the surveyed ICUs

\begin{tabular}{|c|c|c|c|c|c|c|}
\hline & & All & Adult ICUs & & & Pediatric \\
\hline & & & Level I hospitals & Level II hospitals & Level III hospitals & \\
\hline$N$ & - & 73 & 19 & 25 & 16 & 10 \\
\hline Type of ICU & $N(\%)$ & - & - & - & - & - \\
\hline Anesthesiology & - & $30(41.1)$ & $8(42.1)$ & $12(48)$ & $10(62.5)$ & 0 \\
\hline Internal medicine & - & $14(19.2)$ & $4(21.1)$ & $9(36)$ & $1(6.3)$ & 0 \\
\hline Multidisciplinary & - & $13(17.8)$ & $7(36.8)$ & $3(12)$ & $3(18.8)$ & 0 \\
\hline Neurology/neurosurgery & - & $3(4.1)$ & 0 & $1(4)$ & $2(12.5)$ & 0 \\
\hline Pediatrics & - & $2(2.7)$ & 0 & 0 & 0 & $2(2.7)$ \\
\hline Neonatal & - & $8(11)$ & 0 & 0 & 0 & $8(11.1)$ \\
\hline Missing & - & $3(4.1)$ & - & - & - & 0 \\
\hline Number of ICU beds & $N$ & $8(6-12)$ & $7(6-9)$ & $7(6-10)$ & $9(7-12)$ & $12(5-16)$ \\
\hline Level of hospital & $N(\%)$ & - & - & - & - & - \\
\hline Primary care & - & $20(27.4)$ & $19(100)$ & 0 & 0 & $1(10)$ \\
\hline Secondary care & - & $30(41.1)$ & 0 & $25(100)$ & 0 & $5(50)$ \\
\hline Tertiary care & - & $20(27.4)$ & 0 & 0 & $16(100)$ & $4(40)$ \\
\hline Missing & - & $3(4.1)$ & - & - & - & 0 \\
\hline Bed number hospital & $N(\%)$ & - & - & - & - & - \\
\hline$<500$ beds & - & $31(42.5)$ & 18 (94.7) & $5(20)$ & $2(12.5)$ & $3(30)$ \\
\hline $500-1000$ beds & - & $19(26)$ & $1(5.3)$ & $15(60)$ & $1(6.3)$ & $2(20)$ \\
\hline$>1000$ beds & - & $23(31.5)$ & 0 & $5(20)$ & $13(81.3)$ & $5(50)$ \\
\hline ICU architecture & $N(\%)$ & - & - & - & - & - \\
\hline Single bed rooms & - & $15(20.5)$ & $4(21.1)$ & $6(24)$ & $4(25)$ & $1(10)$ \\
\hline Multiple bed rooms & - & $40(54.8)$ & $13(68.4)$ & $15(60)$ & $6(37.5)$ & $6(60)$ \\
\hline Open ICU & - & $14(19.2)$ & $2(10.5)$ & $4(16)$ & $6(37.5)$ & $2(20)$ \\
\hline Missing & - & $4(5.5)$ & - & - & - & $1(10)$ \\
\hline Isolation rooms & $N(\%)$ & $57(78.1)$ & $16(84.2)$ & $23(92)$ & $10(62.5)$ & $8(80)$ \\
\hline Isolation with anteroom & $N(\%)$ & $24(32.9)$ & $4(21.1)$ & $12(48)$ & $3(18.8)$ & $5(50)$ \\
\hline Isolation with air pressure regulation & $N(\%)$ & $22(30.1)$ & $6(31.6)$ & $10(40)$ & $2(12.5)$ & $4(40)$ \\
\hline IMCU adjacent to ICU & $N(\%)$ & $39(53.4)$ & $9(47.4)$ & $16(64)$ & $6(37.5)$ & $8(80)$ \\
\hline Admission of children $<16 y$ rs & $N(\%)$ & - & - & - & - & - \\
\hline Regularly & - & $11(15.1)$ & 0 & $1(4)$ & $1(6.3)$ & $9(90)$ \\
\hline Occasionally & - & $32(43.8)$ & $15(78.9)$ & $10(40)$ & $7(43.8)$ & 0 \\
\hline Never & - & $26(35.6)$ & $4(21.1)$ & $14(56)$ & $8(50)$ & 0 \\
\hline Missing & - & $4(5.5)$ & - & - & - & $1(10)$ \\
\hline
\end{tabular}

dian values with interquartile ranges for continuous variables.

\section{Results}

Of 249 ICUs invited to participate in this survey, 73 questionnaires were completed (return rate of 29.3\%) and included into the statistical analysis. Of these, 60 were reported as adult ICUs and 10 as pediatric including neonatal ICUs. In three questionnaires, the type of ICU and level of hospital the ICU was located in were not reported. Of the remaining adult ICUs, 19, 25 and 16 were located in level I, II and III hospitals, respectively. Table 1 presents characteristics of all surveyed ICUs with data separately reported for adults (categorized according to their location in level I, II and III hospitals) and pediatric ICUs. Of the respondents $78 \%$ reported having isolation rooms available in the ICU with one third stating that room air in isolation rooms can be pressure regulated. Approximately half of the respondents declared that an intermediate care unit was adjacent to the ICU. Staffing characteristics in the surveyed ICUs are detailed in Table 2 and Table 1 of the Electronic Supplementary Material. Of the respondents $89 \%$ reported that the director of their ICU was boardcertified in intensive care medicine. Consultants were given to be constantly present $78 \%$ of ICUs during routine working hours and in $45 \%$ during nights and weekends. The reported nurse:bed ratio varied between 1:1 and 1:2 in 74\% during day shifts and $60 \%$ during night shifts. Routine physiotherapist rounds were reported daily except weekends in $67 \%$ of ICUs. The frequency with which certain monitoring techniques are in use is summarized in Table 3 and Table 2 of the Electronic Supplementary Material. Commonly 
Table 2 Staff characteristics of the surveyed ICUs

\begin{tabular}{|c|c|c|c|c|c|c|}
\hline & & All & Adult ICUs & & & Pediatric \\
\hline & & & Level I hospital & Level II hospital & Level III hospital & \\
\hline$N$ & - & 73 & 19 & 25 & 16 & 10 \\
\hline $\begin{array}{l}\text { ICU director with board-certification in intensive care } \\
\text { medicine }\end{array}$ & $N(\%)$ & $65(89)$ & $19(100)$ & $24(96)$ & $14(87.5)$ & $8(80)$ \\
\hline ICU director $>75 \%$ of work time dedicated to ICU & $N(\%)$ & $44(60.3)$ & $12(63.2)$ & $15(60)$ & $13(81.3)$ & $4(40)$ \\
\hline ICU consultant constantly present on ICU & $N(\%)$ & - & - & - & - & - \\
\hline Regular working hours & - & $57(78.1)$ & $12(63.2)$ & $23(92.0)$ & $14(87.5)$ & $8(80)$ \\
\hline Nights/weekends & - & 33 (45.2) & $9(47.4)$ & $12(48)$ & $11(68.8)$ & $1(10)$ \\
\hline Physician shift patterns & $N(\%)$ & - & - & - & - & - \\
\hline $25 \mathrm{~h}$ shifts & - & $63(86.3)$ & $19(100)$ & $23(92)$ & $13(81.3)$ & $8(80)$ \\
\hline $13 \mathrm{~h}$ shifts & - & $3(4.1)$ & 0 & $2(8)$ & $1(6.3)$ & 0 \\
\hline Missing & - & 7 (9.6) & 0 & 0 & $2(12.5)$ & $2(20)$ \\
\hline Nurse:bed ratio daytime & $N(\%)$ & - & - & - & - & - \\
\hline $1: 1$ & - & $2(2.7)$ & 0 & $1(4)$ & $1(6.3)$ & 0 \\
\hline $1: 1-2$ & - & $30(41.1)$ & $6(31.6)$ & $12(48)$ & $6(37.5)$ & $6(60)$ \\
\hline $1: 2$ & - & $24(32.9)$ & $10(52.6)$ & $8(32)$ & $5(31.3)$ & $1(10)$ \\
\hline $1: 2-3$ & - & $5(6.8)$ & $2(10.5)$ & $1(4)$ & $1(6.3)$ & $1(10)$ \\
\hline $1: 3$ & - & $3(4.1)$ & $1(5.3)$ & $1(4)$ & $1(6.3)$ & 0 \\
\hline Missing & - & $9(12.3)$ & 0 & $2(8)$ & $2(12.5)$ & $2(20)$ \\
\hline Nurse:bed ratio nighttime & $N(\%)$ & - & - & - & - & - \\
\hline $1: 1$ & - & 0 & - & - & - & 0 \\
\hline $1: 1-2$ & - & $9(12.3)$ & $1(5.3)$ & $2(8)$ & $3(18.8)$ & $3(30)$ \\
\hline $1: 2$ & - & $21(28.8)$ & $4(21.1)$ & $11(44)$ & $6(37.5)$ & 0 \\
\hline $1: 2-3$ & - & $23(31.5)$ & $8(42.1)$ & $8(32)$ & $3(18.8)$ & $4(40)$ \\
\hline $1: 3$ & - & $9(12.3)$ & $6(31.6)$ & $1(4)$ & $2(12.5)$ & 0 \\
\hline $1: 3-4$ & - & $2(2.7)$ & 0 & $1(4)$ & 0 & $1(10)$ \\
\hline Missing & - & $9(12.3)$ & 0 & $2(8)$ & $2(12.5)$ & $2(20)$ \\
\hline Nurse shift patterns & $N(\%)$ & - & - & - & - & - \\
\hline $25 \mathrm{~h}$ shifts & - & $2(2.7)$ & 0 & 0 & $2(12.5)$ & 0 \\
\hline $13 \mathrm{~h}$ shifts & - & 64 (87.7) & $19(100)$ & $25(100)$ & $12(75)$ & $8(80)$ \\
\hline Missing & - & $7(9.6)$ & 0 & 0 & $2(12.5)$ & $2(20)$ \\
\hline Physiotherapist rounds & $N(\%)$ & - & - & - & - & - \\
\hline Daily & - & 14 (19.2) & $4(21.1)$ & $6(24)$ & $2(12.5)$ & $2(20)$ \\
\hline Daily except weekends & - & $49(67.1)$ & $13(68.4)$ & $18(72)$ & $12(75)$ & $6(60)$ \\
\hline When needed & - & $3(4.1)$ & $2(10.5)$ & $1(4)$ & 0 & 0 \\
\hline Missing & - & $7(9.6)$ & 0 & 0 & $2(12.5)$ & $2(20)$ \\
\hline
\end{tabular}

used ICU monitoring techniques, such as invasive blood pressure or end-tidal carbon dioxide measurement were reported to be in routine or occasional use in $85 \%$ and $83 \%$ of the ICUs, respectively. Table 4 and Table 3 of the Electronic Supplementary Material display the frequency with which certain therapeutic techniques were used. Patient and family care practices in as well as quality indicators of the surveyed ICUs are summarized in Table 5 and Table 6 as well as Table 4 of the Electronic Supplementary Material, respectively. The majority of ICUs provided a daily visiting time for relatives ranging between 2 and $12 \mathrm{~h}$. Waiting rooms for relatives were available in $68 \%$ of surveyed ICUs. In $66 \%$ of the ICUs, an electronic documentation system was established. Written therapy protocols were available in $70 \%$ of participating ICUs.

\section{Discussion}

This was the first nationwide survey on the structure, organization as well as monitoring and therapeutic capacities of ICUs in Austria. As we could obtain completed questionnaires from only 73 out of 249 invited ICUs, our results cannot be regarded as reflective of the entire cohort of Austrian ICUs but more as a crosssectional overview on the functioning and organizational structures of ICUs in Austria. One reason for the fairly low return rate of $29.3 \%$ might have been that the study period overlapped with the emerging COVID-19 pandemic leading to a premature end of the data collection process. Except for a likely underrepresentation of ICUs located in primary level hospitals, the cohort of ICUs included in our survey ap- 
Table 3 Monitoring techniques available in the surveyed ICUs

\begin{tabular}{|c|c|c|c|c|c|c|}
\hline & & All & Adult ICUs & & & Pediatric \\
\hline & & & Level I hospital & Level II hospital & Level III hospital & \\
\hline$N$ & - & 73 & 19 & 25 & 16 & 10 \\
\hline Invasive blood pressure measurement & $N(\%)$ & - & - & - & - & - \\
\hline Regularly used & - & $60(82.2)$ & $18(94.7)$ & $24(96)$ & $14(87.5)$ & $4(40)$ \\
\hline Occasionally used & - & $2(2.7)$ & 0 & 0 & 0 & $2(20)$ \\
\hline Rarely used & - & $2(2.7)$ & 0 & 0 & 0 & $2(20)$ \\
\hline Never used & - & 0 & 0 & 0 & 0 & 0 \\
\hline Missing & - & $9(12.3)$ & $1(5.3)$ & $1(4)$ & $2(12.5)$ & $2(20)$ \\
\hline End-tidal $\mathrm{CO}_{2}$ measurement & $N(\%)$ & - & - & - & - & - \\
\hline Available at each bed & - & $56(76.7)$ & $17(89.5)$ & $22(88)$ & $13(81.3)$ & $4(40)$ \\
\hline Available at some beds & - & $5(6.8)$ & 0 & $1(4)$ & $1(6.3)$ & $3(30)$ \\
\hline Used on demand & - & $3(4.1)$ & $1(5.3)$ & $1(4)$ & 0 & $1(10)$ \\
\hline Missing & - & $9(12.3)$ & $1(5.3)$ & $1(4)$ & $2(12.5)$ & $2(20)$ \\
\hline Pulse contour analysis use & $N(\%)$ & - & - & - & - & - \\
\hline Regularly used & - & $25(34.2)$ & $10(52.6)$ & $13(52)$ & $2(12.5)$ & 0 \\
\hline Occasionally used & - & $12(16.4)$ & $3(15.8)$ & $3(12)$ & $4(25)$ & $2(20)$ \\
\hline Rarely used & - & $10(13.7)$ & $3(15.8)$ & $5(20)$ & $2(12.5)$ & 0 \\
\hline Never used & - & $17(23.3)$ & $2(10.5)$ & $3(12)$ & $6(37.5)$ & $6(60)$ \\
\hline Missing & - & $9(12.3)$ & $1(5.3)$ & $1(4)$ & $2(12.5)$ & $2(20)$ \\
\hline Transpulmonary thermodilution use & $N(\%)$ & - & - & - & - & - \\
\hline Regularly used & - & $26(35.6)$ & $8(42.1)$ & $14(56)$ & $4(25)$ & 0 \\
\hline Occasionally used & - & $21(28.8)$ & $6(31.6)$ & $8(32)$ & $5(31.3)$ & $2(20)$ \\
\hline Rarely used & - & $8(11)$ & $4(21.1)$ & $1(4)$ & $3(18.8)$ & 0 \\
\hline Never used & - & $9(12.3)$ & 0 & $1(4)$ & $2(12.5)$ & $6(60)$ \\
\hline Missing & - & $9(12.3)$ & $1(5.3)$ & $1(4)$ & $2(12.5)$ & $2(20)$ \\
\hline Pulmonary artery catheter use & $N(\%)$ & - & - & - & - & - \\
\hline Regularly used & - & $7(9.6)$ & 0 & $1(4)$ & $5(31.3)$ & $1(10)$ \\
\hline Occasionally used & - & $20(27.4)$ & $7(36.8)$ & $8(32)$ & $3(18.8)$ & $2(20)$ \\
\hline Rarely used & - & $10(13.7)$ & $2(10.5)$ & $6(24)$ & $2(12.5)$ & 0 \\
\hline Never used & - & $27(37)$ & $9(47.4)$ & $9(36)$ & $4(25)$ & $5(50)$ \\
\hline Missing & - & $9(12.3)$ & $1(5.3)$ & $1(4)$ & $2(12.5)$ & $2(20)$ \\
\hline EEG use in the ICU & $N(\%)$ & - & - & - & - & - \\
\hline Regularly used & - & $29(39.7)$ & $2(10.5)$ & $15(60)$ & $6(37.5)$ & $6(60)$ \\
\hline Occasionally used & - & $24(32.9)$ & $11(57.9)$ & $5(20)$ & $6(37.5)$ & $2(20)$ \\
\hline Rarely used & - & $6(8.2)$ & $2(10.5)$ & $2(8)$ & $2(12.5)$ & 0 \\
\hline Never used & - & $5(6.8)$ & $3(15.8)$ & $2(8)$ & 0 & 0 \\
\hline Missing & - & $9(12.3)$ & $1(5.3)$ & $1(4)$ & $2(12.5)$ & $2(20)$ \\
\hline Intracranial pressure measurement & $N(\%)$ & - & - & - & - & - \\
\hline Regularly used & - & $15(20.5)$ & 0 & $9(36)$ & $6(37.5)$ & 0 \\
\hline Occasionally used & - & $15(20.5)$ & $6(31.6)$ & $2(8)$ & $5(31.3)$ & $2(20)$ \\
\hline Rarely used & - & $4(5.5)$ & 0 & $1(4)$ & $2(12.5)$ & $1(10)$ \\
\hline Never used & - & $30(41.1)$ & $12(63.2)$ & $12(48)$ & $1(6.3)$ & $5(50)$ \\
\hline Missing & - & $9(12.3)$ & $1(5.3)$ & $1(4)$ & $2(12.5)$ & $2(20)$ \\
\hline Mobile $X$-ray available & $N(\%)$ & $64(87.7)$ & $18(94.7)$ & $24(96)$ & $14(87.5)$ & $8(80)$ \\
\hline Sonography available & $N(\%)$ & $64(87.7)$ & $18(94.7)$ & $24(96)$ & $14(87.5)$ & $8(80)$ \\
\hline Transthoracic echocardiography use & $N(\%)$ & - & - & - & - & - \\
\hline Regularly used & - & $59(80.8)$ & $16(84.2)$ & $23(92)$ & $12(75)$ & $8(80)$ \\
\hline Occasionally used & - & $2(2.7)$ & $1(5.3)$ & 0 & $1(6.3)$ & 0 \\
\hline Rarely used & - & $3(4.1)$ & $1(5.3)$ & $1(4)$ & $1(6.3)$ & 0 \\
\hline Never used & - & 0 & 0 & 0 & 0 & 0 \\
\hline
\end{tabular}


Table 3 (Continued)

\begin{tabular}{|c|c|c|c|c|c|c|}
\hline & & All & Adult ICUs & & & Pediatric \\
\hline & & & Level I hospital & Level II hospital & Level III hospital & ICUs \\
\hline Missing & - & $9(12.3)$ & $1(5.3)$ & $1(4)$ & $2(12.5)$ & $2(20)$ \\
\hline Transesophageal echocardiography use & $N(\%)$ & - & - & - & - & - \\
\hline Regularly used & - & $38(52.1)$ & $10(52.6)$ & $18(72)$ & $10(62.5)$ & 0 \\
\hline Occasionally used & - & $13(17.8)$ & $4(21.1)$ & $4(16)$ & $3(18.8)$ & $2(20)$ \\
\hline Rarely used & - & $7(9.6)$ & $4(21.1)$ & $2(8)$ & $1(6.3)$ & 0 \\
\hline Never used & - & $6(8.2)$ & 0 & 0 & 0 & $6(60)$ \\
\hline Missing & - & $9(12.3)$ & $1(5.3)$ & $1(4)$ & $2(12.5)$ & $2(20)$ \\
\hline Blood gas analyzer use & $N(\%)$ & - & - & - & - & - \\
\hline Regularly used & - & $64(87.7)$ & $18(94.7)$ & $24(96)$ & $14(87.5)$ & $8(80)$ \\
\hline Occasionally used & - & 0 & 0 & 0 & 0 & 0 \\
\hline Rarely used & - & 0 & 0 & 0 & 0 & 0 \\
\hline Never used & - & 0 & 0 & 0 & 0 & 0 \\
\hline Missing & - & $9(12.3)$ & $1(5.3)$ & $1(4)$ & $2(12.5)$ & $2(20)$ \\
\hline Thromboelastometry use & $N(\%)$ & - & - & - & - & - \\
\hline Regularly used & - & $32(43.8)$ & $11(57.9)$ & $16(64)$ & $5(31.3)$ & 0 \\
\hline Occasionally used & - & $10(13.7)$ & $3(15.8)$ & 1 (4) & $5(31.3)$ & $1(10)$ \\
\hline Rarely used & - & $8(11)$ & $2(10.5)$ & $2(8)$ & $3(18.8)$ & $1(10)$ \\
\hline Never used & - & 14 (19.2) & $2(10.5)$ & $5(20)$ & $1(6.3)$ & $6(60)$ \\
\hline Missing & - & $9(12.3)$ & $1(5.3)$ & $1(4)$ & $2(12.5)$ & $2(20)$ \\
\hline
\end{tabular}

pears well balanced between adult and pediatric ICUs as well as ICUs located in secondary and tertiary level hospitals. As mentioned before, this survey did not aim at evaluating details of ICUs that are regulated and determined by the Austrian Health Care Strucutre Plan (Österreichischer Strukturplan Gesundheit [4]). Accordingly, instead of evaluating whether certain diagnostic or therapeutic equipment was available, we sought to determine how frequently these techniques were in use in order to gain insights into the current practice of ICU care in Austria. This is also the reason why the results of this survey neither intended to nor can evaluate whether regulatory requirements were met by the surveyed ICUs.

The median number of beds in the ICUs included in our survey was eight. Over three quarters of the responding ICU directors stated that their ICUs were architectonically arranged in multiple bed rooms or open ICUs (i.e., ICU halls). This is in contrast to modern ICU design which currently focuses on single rooms to optimize patient privacy and allow for undisturbed patient-family interaction [6]. Isolation rooms were reported to be available in $78.1 \%$ of surveyed ICUs. This number appears remarkably high but could be the result of a lacking uniform definition of what an isolation room is (e.g. some respondents may have referred to single patient rooms as isolation rooms); however, only one third of the ICUs enrolled in this analysis had an isolation room with an anteroom and/or the possibility to regulate air pressure in the isolation room. In view of the current SARS$\mathrm{CoV}-2$ pandemic and preparedness for upcoming care of critically ill patients suffering from highly contagious infectious diseases, it appears advisable that isolation facilities should routinely be included into the planning of future ICUs in Austria.

Both the number of consultant-led ICU services as well as the nurse:bed ratio among the survey ICUs were high compared to reports from other countries $[7,8]$. Staffing patterns of ICU physicians and nurses have been associated with survival of critically ill patients $[8,9]$. Multidisciplinary structures are a key feature of today's intensive care medicine. This is also reflected by the results of our survey. Physiotherapists and psychologists were found to be the medical partners most frequently involved in the care of critically ill patients in addition to the ICU team. In contrast to ICU practice in other regions of the world, particularly in Anglo-American countries, other medical specialists (e.g., radiologists, infectious disease specialists, palliative care teams) and professions (e.g., pharmacists, dieticians) were not reported to be routinely involved in patient care in the surveyed ICUs. These partners were consulted in an on-demand fashion. Published evidence suggests that involvement of other medical specialists and professions has the potential to improve patient care, safety and outcomes in the ICU [10-12].

Our results on the frequency of use of diagnostic and therapeutic techniques clearly highlight that echocardiography is a regularly used diagnostic technique in the vast majority of surveyed ICUs. This finding is in line with guidelines underlining the rapid and high diagnostic yield of bedside echocardiogra- 
Table 4 Therapeutic techniques available in the surveyed ICUs

\begin{tabular}{|c|c|c|c|c|c|c|}
\hline & & \multirow[t]{2}{*}{ All } & \multicolumn{3}{|l|}{ Adult ICUs } & \multirow{2}{*}{$\begin{array}{l}\text { Pediatric } \\
\text { ICUs }\end{array}$} \\
\hline & & & Level I hospital & Level II hospital & Level III hospital & \\
\hline$N$ & - & 73 & 19 & 25 & 16 & 10 \\
\hline High-flow nasal oxygen therapy & $N(\%)$ & - & - & - & - & - \\
\hline Regularly used & - & $58(79.5)$ & $15(78.9)$ & $21(84)$ & $14(87.5)$ & $8(80)$ \\
\hline Occasionally used & - & $3(4.1)$ & $2(10.5)$ & $1(4)$ & 0 & 0 \\
\hline Rarely used & - & $2(2.7)$ & $1(5.3)$ & $1(4)$ & 0 & 0 \\
\hline Never used & - & $1(1.4)$ & 0 & $1(4)$ & 0 & 0 \\
\hline Missing & - & $9(12.3)$ & $1(5.3)$ & $1(4)$ & $2(12.5)$ & $2(20)$ \\
\hline Noninvasive ventilation & $N(\%)$ & - & - & - & - & - \\
\hline Regularly used & - & $61(83.6)$ & 17 (89.5) & $23(92)$ & $13(81.3)$ & $8(80)$ \\
\hline Occasionally used & - & $1(1.4)$ & 0 & $1(4)$ & 0 & 0 \\
\hline Rarely used & - & $2(2.7)$ & $1(5.3)$ & 0 & $1(6.3)$ & 0 \\
\hline Never used & - & 0 & 0 & 0 & 0 & 0 \\
\hline Missing & - & $9(12.3)$ & $1(5.3)$ & $1(4)$ & $2(12.5)$ & $2(20)$ \\
\hline Invasive ventilation & $N(\%)$ & - & - & - & - & - \\
\hline Regularly used & - & $61(83.6)$ & 17 (89.5) & $24(96)$ & $14(87.5)$ & $6(60)$ \\
\hline Occasionally used & - & $1(1.4)$ & 0 & 0 & 0 & $1(10)$ \\
\hline Rarely used & - & $2(2.7)$ & $1(5.3)$ & 0 & 0 & $1(10)$ \\
\hline Never used & - & 0 & 0 & 0 & 0 & 0 \\
\hline Missing & - & $9(12.3)$ & $1(5.3)$ & $1(4)$ & $2(12.5)$ & $2(20)$ \\
\hline Percutaneous tracheostomy & $N(\%)$ & - & - & - & - & - \\
\hline Regularly used & - & $28(38.4)$ & $7(36.8)$ & $11(44)$ & $10(62.5)$ & 0 \\
\hline Occasionally used & - & $14(19.2)$ & $6(31.6)$ & $3(12)$ & $1(6.3)$ & $4(40)$ \\
\hline Rarely used & - & $12(16.4)$ & $3(15.8)$ & $6(24)$ & $3(18.8)$ & 0 \\
\hline Never used & - & $10(13.7)$ & $2(10.5)$ & $4(16)$ & 0 & $4(40)$ \\
\hline Missing & - & $9(12.3)$ & $1(5.3)$ & $1(4)$ & $2(12.5)$ & $2(20)$ \\
\hline CVWHDF/CVHF & $N(\%)$ & - & - & - & - & - \\
\hline Regularly used & - & $46(63)$ & 15 (78.9) & $20(80)$ & 11 (68.8) & 0 \\
\hline Occasionally used & - & $3(4.1)$ & 0 & $3(12)$ & 0 & 0 \\
\hline Rarely used & - & $4(5.5)$ & $2(10.5)$ & $1(4)$ & 0 & $1(10)$ \\
\hline Never used & - & $11(15.1)$ & $1(5.3)$ & 0 & $3(18.8)$ & $7(70)$ \\
\hline Missing & - & $9(12.3)$ & $1(5.3)$ & $1(4)$ & $2(12.5)$ & $2(20)$ \\
\hline Hemodialysis & $N(\%)$ & - & - & - & - & - \\
\hline Regularly used & - & $21(28.8)$ & $6(31.6)$ & $11(44)$ & $4(25)$ & 0 \\
\hline Occasionally used & - & $6(8.2)$ & $1(5.3)$ & $4(16)$ & $1(6.3)$ & 0 \\
\hline Rarely used & - & $7(9.6)$ & $2(10.5)$ & $3(12)$ & $2(12.5)$ & 0 \\
\hline Never used & - & $30(41.1)$ & $9(47.4)$ & $6(24)$ & $7(43.8)$ & $8(80)$ \\
\hline Missing & - & $9(12.3)$ & $1(5.3)$ & $1(4)$ & $2(12.5)$ & $2(20)$ \\
\hline Veno-venous ECMO & $N(\%)$ & - & - & - & - & - \\
\hline Regularly used & - & $11(15.1)$ & 0 & $5(20)$ & $6(37.5)$ & 0 \\
\hline Occasionally used & - & $6(8.2)$ & 0 & $6(24)$ & 0 & 0 \\
\hline Rarely used & - & $1(1.4)$ & $1(5.3)$ & 0 & 0 & 0 \\
\hline Never used & - & $46(63)$ & 17 (89.5) & $13(52)$ & $8(50)$ & $8(80)$ \\
\hline Missing & - & $9(12.3)$ & $1(5.3)$ & $1(4)$ & $2(12.5)$ & $2(20)$ \\
\hline Veno-arterial ECMO & $N(\%)$ & - & - & - & - & - \\
\hline Regularly used & - & $10(13.7)$ & 0 & $4(16)$ & $6(37.5)$ & 0 \\
\hline Occasionally used & - & $1(1.4)$ & 0 & $1(4)$ & 0 & 0 \\
\hline Rarely used & - & $1(1.4)$ & 0 & $1(4)$ & 0 & 0 \\
\hline Never used & - & $52(71.2)$ & 18 (94.7) & $18(72)$ & $8(50)$ & $8(80)$ \\
\hline Missing & - & $9(12.3)$ & $1(5.3)$ & $1(4)$ & $2(12.5)$ & $2(20)$ \\
\hline
\end{tabular}

$\mathrm{CO}_{2}$ carbon dioxide, $\mathrm{CVVHDF} / C V H F$ continuous veno-venous hemodiafiltration/hemofiltration, ECMO extracorporeal membrane oxygenation, ICU intensive care unit 
Table 5 Patient and family care practices in the surveyed ICUs

\begin{tabular}{|c|c|c|c|c|c|c|}
\hline & & All & Adult ICUs & & & Pediatric \\
\hline & & & Level I hospital & Level II hospital & Level III hospital & \\
\hline$N$ & - & 73 & 19 & 25 & 16 & 10 \\
\hline Visiting hours & $N(\%)$ & - & - & - & - & - \\
\hline$<2$ & - & $2(2.7)$ & 0 & $2(8)$ & 0 & 0 \\
\hline $2-4$ & - & $21(28.8)$ & $3(15.8)$ & $9(36)$ & $9(56.3)$ & 0 \\
\hline $4-6$ & - & $16(21.9)$ & $6(31.6)$ & $5(20)$ & $4(25)$ & $1(10)$ \\
\hline $6-12$ & - & $10(13.7)$ & $6(31.6)$ & $3(12)$ & $1(6.3)$ & 0 \\
\hline $12-24$ & - & $6(8.2)$ & 0 & $2(8)$ & 0 & $4(40)$ \\
\hline 24 & - & $8(11)$ & $3(15.8)$ & $2(8)$ & 0 & $3(30)$ \\
\hline Missing & - & $10(13.7)$ & $1(5.3)$ & $2(8)$ & $2(12.5)$ & $2(20)$ \\
\hline Waiting room for relatives & $N(\%)$ & $50(68.5)$ & $15(78.9)$ & $18(72)$ & $9(56.3)$ & $8(80)$ \\
\hline Separate room for family discussions & $N(\%)$ & $44(60.3)$ & $11(57.9)$ & $18(72)$ & $8(50)$ & $7(70)$ \\
\hline Psychologist support & $N(\%)$ & - & - & - & - & - \\
\hline Daily & - & $28(38.4)$ & $9(47.4)$ & $10(40)$ & $5(31.3)$ & $4(40)$ \\
\hline Daily except weekends & - & $19(26)$ & $5(26.3)$ & $9(36)$ & $2(12.5)$ & $3(30)$ \\
\hline Some days & - & $5(6.8)$ & $1(5.3)$ & $1(4)$ & $3(18.8)$ & 0 \\
\hline When needed & - & $11(15.1)$ & $3(15.8)$ & $3(12)$ & $4(25)$ & $1(10)$ \\
\hline Never & - & 0 & 0 & 0 & 0 & 0 \\
\hline Missing & - & $10(13.7)$ & $1(5.3)$ & $2(8)$ & $2(12.5)$ & $2(20)$ \\
\hline Information brochure for families & $N(\%)$ & - & - & - & - & - \\
\hline In German and other languages & - & $27(37)$ & $4(21.1)$ & $8(32)$ & $9(56.3)$ & $6(60)$ \\
\hline Only in German & - & $28(38.4)$ & $12(63.2)$ & $11(44)$ & $3(18.8)$ & $2(20)$ \\
\hline Unavailable & - & $8(11)$ & $2(10.5)$ & $4(16)$ & $2(12.5)$ & 0 \\
\hline Missing & - & $10(13.7)$ & $1(5.3)$ & $2(8)$ & $2(12.5)$ & $2(20)$ \\
\hline ICU diary for long-term patients & $N(\%)$ & $16(21.9)$ & $2(10.5)$ & $7(28)$ & $3(18.8)$ & $4(40)$ \\
\hline Post-ICU follow-up care of long-term patients & $N(\%)$ & 14 (19.2) & $3(15.8)$ & $3(12)$ & $1(6.3)$ & $7(70)$ \\
\hline
\end{tabular}

phy and ultrasound in the ICU [13, 14]. Similarly, electroencephalography was regularly or occasionally used in approximately three quarters of the surveyed ICUs. This likely mirrors the usefulness of electroencephalography to predict neurological outcome and the evolving understanding that nonconvulsive status epilepticus may mimic hypoactive delirium in ICU patients [15]. While extracorporeal life support appears to be used on a regular base in selected centers, artificial liver support was found to be used in only one of the ICUs included in this survey (Table 3 of the Electronic Supplementary Material). It is also noteworthy that some therapeutic (e.g., nitric oxide inhalation, high-frequency oscillatory ventilation, extracorporeal blood purification techniques, intra-aortic balloon counterpulsation) or diagnostic (e.g., near infrared spectroscopy) techniques, for which only limited evidence or even evidence against its standard use exists [16-21], were found to be regularly used in up to one quarter of ICUs in this cohort (Table 3 of the Electronic Supplementary Material).

Our survey also evaluated selected quality indicators of critical care in Austrian ICUs. Some of these quality indicators (e.g., goals of therapy, sedation depth, pain scale, conversations with relatives and therapy limitations) were reported to be systemati- cally documented in most ICUs. On the other hand, some quality indicators, such as those recommended by the European Society of Intensive Care Medicine [22] and the German Interdisciplinary Association of Intensive and Emergency Medicine [23], were found to be documented in only half of the surveyed ICUs. Similarly, only half of the respondents reported that written management protocols were available for certain procedures or selected pathologies. While less than half of the respondents stated that the ICU participated in a national or international benchmarking project, more than two thirds of respondents expressed interest in taking part in such a benchmarking project.

Many of the differences between adult and pediatric ICUs observed in our survey (e.g., differences in the use of monitoring techniques and treatment) can be explained by the dissimilar patient populations cared for in adult and pediatric ICUs. On the other hand, we also identified differences uninfluenced by the age of ICU patients. For example, respondents from pediatric ICUs reported more liberal visiting hours than those from adult ICUs. Several publications have indicated that more liberal or even open visiting hours, where family members or selected support persons have unrestricted access to the critically 
Table 6 Quality indicators of the surveyed ICUs

\begin{tabular}{|c|c|c|c|c|c|c|}
\hline & & All & Adult ICUs & & & Pediatric \\
\hline & & & Level I hospitals & Level II hospitals & Level III hospitals & \\
\hline$N$ & - & 73 & 19 & 25 & 16 & 10 \\
\hline Hospital-wide ICU bed coordination & $N(\%)$ & $25(34.2)$ & $2(10.5)$ & $12(48)$ & $6(37.5)$ & $5(50)$ \\
\hline SOP defining ICU admission & $N(\%)$ & $24(32.9)$ & $8(42.1)$ & $10(40)$ & $2(12.5)$ & $4(40)$ \\
\hline SOP defining ICU discharge & $N(\%)$ & $26(35.6)$ & $9(47.4)$ & $9(36)$ & $4(25)$ & $4(40)$ \\
\hline Hospital-wide cardiac arrest team & $N(\%)$ & $59(80.8)$ & $16(84.2)$ & $22(88)$ & $14(87.5)$ & $7(70)$ \\
\hline Hospital-wide medical emergency team & $N(\%)$ & $28(38.4)$ & $7(36.8)$ & $11(44)$ & $4(25)$ & $6(60)$ \\
\hline Electronic documentation in ICU (PDMS) & $N(\%)$ & $48(65.8)$ & $9(47.4)$ & $20(80)$ & $13(81.3)$ & $6(60)$ \\
\hline Documentation of goals of therapy & $N(\%)$ & $56(76.7)$ & $16(84.2)$ & $21(84)$ & $13(81.3)$ & $6(60)$ \\
\hline Daily documentation of depth of sedation & $N(\%)$ & $55(75.3)$ & 17 (89.5) & $22(88)$ & $13(81.3)$ & $3(30)$ \\
\hline Daily documentation of pain scale & $N(\%)$ & $59(80.8)$ & $18(94.7)$ & $22(88)$ & $13(81.3)$ & $6(60)$ \\
\hline Daily screening for delirium & $N(\%)$ & $41(56.2)$ & $16(84.2)$ & $12(48)$ & $12(75)$ & $1(10)$ \\
\hline Documentation of conversations with relatives & $N(\%)$ & $47(64.4)$ & $16(84.2)$ & $16(64)$ & $8(50)$ & $7(70)$ \\
\hline Documentation of therapy limitations & $N(\%)$ & $63(86.3)$ & $18(94.7)$ & $23(92)$ & $14(87.5)$ & $8(80)$ \\
\hline $\begin{array}{l}\text { Hospital-wide availability of a critical incident reporting } \\
\text { system }\end{array}$ & $N(\%)$ & $56(76.7)$ & $16(84.2)$ & $21(84)$ & $13(81.3)$ & $6(60)$ \\
\hline Regular M\&M conferences & $N(\%)$ & $42(57.5)$ & $12(63.2)$ & $14(56)$ & $9(56.3)$ & $7(70)$ \\
\hline DIN/DIVI-based color coding of drugs & $N(\%)$ & $46(63)$ & $15(78.9)$ & $18(72)$ & $8(50)$ & $5(50)$ \\
\hline $\begin{array}{l}\text { Participation in national/international benchmarking } \\
\text { projects }\end{array}$ & $N(\%)$ & $35(47.9)$ & $8(42.1)$ & $16(64)$ & $6(37.5)$ & $5(50)$ \\
\hline Interested to participate in national benchmarking projects & $N(\%)$ & $51(69.9)$ & $13(68.4)$ & $20(80)$ & $13(81.3)$ & $5(50)$ \\
\hline Availability of written therapy protocols & $N(\%)$ & $51(69.9)$ & $14(73.7)$ & $22(88)$ & $8(50)$ & $7(70)$ \\
\hline
\end{tabular}

ill patient, do not only support the concept of patientcentered care but have also been associated with improved family satisfaction and patient outcomes in adult ICUs [24]. Another interesting finding was that respondents from pediatric ICUs more often reported that an intermediate care unit was adjacent to their ICU than respondents from adult ICUs $(80 \%$ vs. $51.7 \%$ ). Intermediate care units not only avoid ICU admission but also facilitate a safe step-down from the ICU to the general ward in high-risk patients [25, 26]. Furthermore, while $70 \%$ of respondents from pediatric ICUs reported to follow-up long-term patients after ICU discharge, only a minority (11.7\%) of respondents from adult ICUs did so. Although to date the longitudinal care model of outpatient follow-up after ICU discharge is not yet evidence-based, positive effects on patient and family experiences as well as improvement of ICU quality have been reported [27].

Certain limitations need to be considered when interpreting the results of this survey. First, we cannot exclude that a selection bias has occurred and influenced our survey results. Furthermore, it is a distinctive limitation of questionnaire-based surveys that opinions rather than the true clinical practice are collected [28]. Therefore, although our survey mostly focused on structural and objective data, we cannot verify that the information provided by the respondents reflects the actual situation in the surveyed ICUs.
In conclusion, the Austrian ICU survey suggests that ICUs in Austria are clearly structured, well-organized and well-equipped and have a high nurse:bed ratio. In view of the relatively low return rate, we cannot exclude that a selection bias has led to overestimation of the survey findings.

Acknowledgements The study investigators wish to thank all member societies of the Federation of Austrian Societies of Intensive Care Medicine (FASIM) and respondents for their support of the Austrian ICU survey.

Conflict of interest C. Schlömmer, G.A. Schittek, J. Meier, W. Hasibeder, A. Valentin and M.W. Dünser declare that they have no competing interests.

\section{References}

1. Rhodes A, Ferdinande P, Flaatten H, et al. The variability of critical care bed numbers in Europe. Intensive Care Med. 2012;38(10):1647-53.

2. Vincent JL, Sakr Y, Sprung CL, et al. Sepsis in European intensive care units: results of the SOAP study. Crit Care Med. 2006;34(2):344-53.

3. OECD. Intensive care beds capacity. 2020. https://www. oecd.org/coronavirus/en/data-insights/intensive-carebeds-capacity. Accessed 10 Apr 2021.

4. Bundesministerium für Soziales, Gesundheit, Pflege und Konsumentenschutz. Österreichischer Sturkturplan Gesundheit. 2017. https:/goeg.at/sites/goeg.at/files/ 
inline-files/2 \%C3\%96SG_2017_Textband_Stand_18.12. 2020.pdf. Accessed 12.2020 .

5. Joannidis M, Klein SJ, Metnitz P, Valentin A. Vergütung intensivmedizinischer Leistungen in Österreich. Einsatz des LKF-Systems. Med Klin Intensivmed Notfmed. 2018;113:28-32.

6. Jongerden IP, Slooter AJ, Peelen LM, et al. Effect of intensive care environment on family and patient satisfaction: a before-after study. Intensive Care Med. 2013;39(9):1626-34.

7. Graf J, Reinhold A, Brunkhorst FM, et al. Variability of structures in German intensive care units-a representative, nationwide analysis. Wien Klin Wochenschr. 2008;122(19-20):572-8.

8. Ward NS, Afessa B, Kleinpell R, et al. Intensivist/patient ratios in closed ICUs: a statement from the society of critical care medicine taskforce on ICU staffing. Crit Care Med. 2013;41(2):638-45.

9. West E, Barron DN, Harrison D, Rafferty AM, Rowan K, Sanderson C. Nurse staffing, medical staffing and mortality in intensive care: an observational study. Int J Nurs Stud. 2014;51(5):781-94.

10. Gilbert DN. Influence of an infectious diseases specialist on ICU multidisciplinary rounds. Crit Care Res Pract. 2014;2014:307817.

11. Soguel L, RevellyJP, Schaller MD, Longchamp C, Berger MM. Energy deficit and length of hospital stay can be reduced by a two-step quality improvement of nutrition therapy: the intensive care unit dietitian can make the difference. Crit Care Med. 2012;40(2):412-9.

12. MacLaren R, Bond CA. Effects of pharmacist participation in intensive care units on clinical and economic outcomes of critically ill patients with thromboembolic or infarctionrelated events. Pharmacotherapy. 2009;29(7):761-8.

13. Frankel HL, Kirkpatrick AW, Elbarbary M, et al. Guidelines for the appropriate use of bedside general and cardiac ultrasonography in the evaluation of critically ill patients-part I: general ultrasonography. Crit Care Med. 2015;43(11):2479-502.

14. Levitov A, Frankel HL, Blaivas M, et al. Guidelines for the appropriate use of bedside general and cardiac ultrasonography in the evaluation of critically ill patients-part II: cardiac ultrasonography. Crit Care Med. 2016;44(6):1206-27.

15. Claassen J, Taccone FS, Horn P, Holtkamp M, Stocchetti N, Oddo M. Neurointensive care section of the European society of intensive care medicine. Recommendations on the use of EEG monitoring in critically ill patients: consensus statement from the neurointensive care section of the ESICM. Intensive Care Med. 2013;39(8):1337-51.

16. Karam O, Gebistorf F, Wetterslev J, Afshari A. The effect of inhaled nitric oxide in acute respiratory distress syndrome in children and adults: a Cochrane systematic review with trial sequential analysis. Anaesthesia. 2017;72(1):106-17.

17. Ferguson ND, Cook DJ, Guyatt GH, et al. High-frequency oscillation in early acute respiratory distress syndrome. NEngl J Med. 2013;368(9):795-805.

18. Schittek GA, Zoidl P, Eichinger M, et al. Adsorption therapy in critically ill with septic shock and acute kidney injury: a retrospective and prospective cohort study. Ann Intensive Care. 2020;10:154.

19. Snow TAC, Littlewood S, Corredor C, Singer M, Asulkumaran N. Effect of extracorporeal blood purification on mortality in sepsis: a meta-analysis and trial sequential analysis. Blood Purif. 2020;28:1-11.

20. Thiele H, Zeymer U, Neumann FJ, et al. Intraaortic balloon support for myocardial infarction with cardiogenic shock. NEngl J Med. 2012;367(14):1287-96.

21. Lewis C, Hogue CW. Lack of benefit of near-infrared spectroscopy monitoring for improving patient outcomes. Case closed? BrJAnaesth. 2017;119(3):347-9.

22. Rhodes A, Moreno RP, AzoulayE, etal. Prospectively defined indicators to improve the safety and quality of care for critically ill patients: a report from the task force on safety and quality of the European society of intensive care medicine (ESICM). Intensive Care Med. 2012;38(4):598-605.

23. KumpfO, Braun JP, Brinkmann A, et al. Quality indicators in intensive care medicine for Germany-third edition 2017. GerMed Sci. 2017;15:Doc10.

24. Whitton S, Pittiglio LI. Critical care open visiting hours. Crit Care Nurse. 2011;34(4):361-6.

25. Lekwijit S, Chan CW, Green LV, Liu VX, Escobar GJ. The impact of step-down unit care on patient outcomes after ICU discharge. Crit Care Explor. 2020;2(5):e114.

26. Franklin CM, Rackow EC, Mamdani B, Nightingale S, Burke G, Weil MH. Decreases in mortality on a large urban medical service by facilitating access to critical care. An alternative to rationing. Arch Intern Med. 1988;148(6):1403-5.

27. Williams TA, Leslie GD. Beyond the walls: a review of ICU clinics and their impact on patient outcomes after leaving hospital. Aust Crit Care. 2008;21(1):6-17.

28. BrunkhorstF, EngelC, Ragaller M, etal. Practice and perception-a nationwide survey of therapy habits in sepsis. Crit Care Med. 2008;36(10):2719-25.

Publisher's Note Springer Nature remains neutral with regard to jurisdictional claims in published maps and institutional affiliations. 